\title{
Estimativa do conteúdo de nutrientes em um povoamento jovem de Acacia mearnsii De Wild. estabelecido na região sul do Brasil
}

\author{
Marcos Vinicius Winckler CALdeira ${ }^{(1)}$ \\ Mauro V ALdir Schumacher ${ }^{(2)}$ \\ Elias Moreira Dos SAnTOS $^{(3)}$ \\ Neura Tedesco ${ }^{(4)}$ \\ Josani Carbonera Pereira ${ }^{(5)}$
}

\section{RESUMO}

No presente trabalho foi quantificado o conteúdo de nutrientes em uma procedência australiana de Lake Geoge Bunge Dore, acácia-negra, (Acacia mearnsii De Wild.), aos 2,4 anos de idade. Esse material encontra-se estabelecido em solos de baixa fertilidade, com acidez elevada, localizado na Fazenda Menezes, no Distrito de Capão Comprido, município de Butiá, RS. Foram selecionados um total de 9 árvores para comporem as amostras. A amostragem constituiu na individualização dos compartimentos da biomassa aérea (folhas, galhos vivos, galhos mortos, casca e madeira) visando à determinação da matéria seca e do conteúdo de nutrientes. As quantidades de nutrientes contidas na biomassa total desse material foram de $334,37 \mathrm{~kg} \cdot \mathrm{ha}^{-1}$ de N; 23,65 kg.ha ${ }^{-1}$ de P; 259,67 kg.ha ${ }^{-1}$ de K; 107,73 kg.ha ${ }^{-1}$ de Ca; 35,09 kg.ha ${ }^{-1}$ de $\mathrm{Mg}$ e 21,43 $\mathrm{kg} \mathrm{ha}^{-1}$ de S. Quanto a distribuição nos compartimentos, 61,07\% da matéria seca foi alocada para as folhas e galhos (vivos e mortos), contento $73 \%$ do N; $72,4 \%$ do P; $64 \%$ do $\mathrm{K} ; 55 \%$ do $\mathrm{Ca}, 66 \%$ do $\mathrm{Mg}$ e $75 \%$ do S do total existente na parte aérea. $\mathrm{O}$ componente fuste (casca e madeira) acumulou $27 \%$ do N; $27,6 \%$ do P; $36 \%$ do K; $45 \%$ do Ca, $34 \%$ do Mg e $25 \%$ do S.

\footnotetext{
${ }^{1}$ Eng. Florestal, Doutorando em Eng. Florestal, Curso de Pós-graduação em Eng. Florestal/UFPR. Centro de Ciências Florestais e da Madeira. Rua Pref. Lothário Meissner, 3400 - Jardim Botânico; 80210-170. Curitiba, PR. CREA nº 87023-D/RS. caldeira@ floresta.ufpr.br .

2 Eng. Florestal, Doutor, Prof. Adjunto, CCR/UFSM. Santa Maria/RS. SIAPE: 2173941

${ }^{3}$ Eng. Florestal, Sup. de Pesquisa e Desenvolvimento da AGROSETA S.A. CREA no 83648-D/RS

4 Eng. Florestal, Mestre em Engenharia Florestal. Rua 2, no 115; Parque Residencial Santa Lúcia Camobi; CEP: 97105-900. Santa Maria/RS. (OXX) 55226 3154. CREA n 90276-D/RS

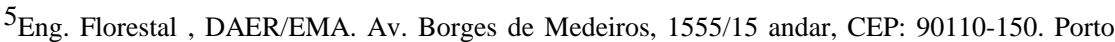
Alegre/RS. CREA: 93295-D
} 
Palavras-chave: nutrientes minerais, Acacia mearnsii, ciclagem de nutrientes, sustentabilidade, procedência

\section{ABSTRAT \\ Estimation of content of nutrients in a young stand of Acacia} mearnsii De Wild. established in the southern of Brazil. The content of nutrients in a black wattle australian Lake George Bunge Dore provenance (Acacia mearnsii De Wild.) with 2.4 years old was quantified. The stands are established in low fertility soils with high acidity at Menezes Farm, Capão Comprido District, municipality of Butiá, RS. Nine trees were selected to compound the samples. The sampling consisted on separating the above-ground biomass compartments (leaves, live branches, dead branches, bark and wood) to determine the dry matter and the nutrient contents. The nutrient quantities in the above-ground biomass were $334.37 \mathrm{~kg} \cdot \mathrm{ha}^{-1} \mathrm{~N} ; 23.65 \mathrm{~kg} \cdot \mathrm{ha}^{-1} \mathrm{P} ; 259.67$ kg.ha ${ }^{-1} \mathrm{~K} ; 107.73 \mathrm{~kg} \cdot \mathrm{ha}^{-1} \mathrm{Ca} ; 35,09 \mathrm{~kg} \cdot \mathrm{ha}^{-1} \mathrm{Mg}$ and $21.43 \mathrm{~kg} \mathrm{ha}^{-1}$ de S. $61.07 \%$ of dry matter was allocated to leaves and branches (live and dead) containing $73 \% \mathrm{~N} ; 72.4 \% \mathrm{P} ; 64 \% \mathrm{~K} ; 55 \% \mathrm{Ca}, 66 \% \mathrm{Mg}$ and $75 \% \mathrm{~S}$. The stem component (bark and wood) accumulated $27 \% \mathrm{~N} ; 27.6 \% \mathrm{P} ; 36 \% \mathrm{~K} ; 45 \% \mathrm{Ca}, 34 \% \mathrm{Mg}$ and $25 \% \mathrm{~S}$.

Key words: mineral nutrients, black wattle, nutrient cycling, sustainability, provenance.

\section{INTRODUÇÃO}

Considerando-se as florestas do mundo como um todo, verifica-se que o estoque de nutrientes na vegetação aumenta das florestas boreais para as tropicais. Por outro lado, a massa de nutrientes acumulados na serapilheira aumenta de forma contrária, ou seja, das florestas tropicais para as boreais. Isto ocorre, principalmente, devido à baixa atividade dos organismos decompositores, que são inibidos pelas baixas temperaturas (KIMMINS, 1987).

Segundo PRITCHETT (1990), a absorção dos nutrientes pelas árvores é influenciada pela espécie, pela cobertura do dossel e pelas condições edafoclimáticas. Em princípio, a absorção anual de nutrientes pelas árvores é da mesma ordem das apresentadas pelas culturas agrícolas mas, como a maior parte dos nutrientes absorvidos é devolvida ao piso florestal, quantidades relativamente pequenas são retidas no acréscimo anual da biomassa arbórea.

A quantidade de nutrientes, num ecossistema florestal, é representada pelo somatório dos nutrientes contidos nos diferentes componentes da biomassa arbórea, vegetação do sub-bosque, serapilheira e solo (POGGIANI, 1992). 
O acúmulo de nutrientes na biomassa arbórea, segundo SCHUMACHER (1992), varia conforme o elemento, do solo, e características nutricionais de cada espécie e da idade da floresta.

De acordo com VAN DEN DRIESSCHE (1984), as coníferas tendem a ter maior proporção de biomassa foliar do que as folhosas decíduas. A maior proporção do conteúdo total de nutrientes na árvore é encontrada na folhagem de coníferas (20 a $25 \%$ ) do que em folhosas decíduas (8 a 10\%), ainda que a concentração de nutrientes nas folhas de coníferas seja mais baixa do que nas folhosas (RODIN \& BAZILEVICH, 1967).

Principalmente nas regiões tropicais e subtropicais é fundamental que se tenha conhecimentos sobre a dinâmica dos nutrientes nos diversos compartimentos de um ecossistema florestal, para que se possa adotar um manejo que assegure a sustentabilidade (SCHUMACHER, 1996). Portanto, o presente estudo teve por objetivo quantificar o conteúdo de nutrientes na biomassa aérea em um povoamento de acácia-negra (Acacia mearnsii) de procedência Lake George Bunge Dore, Austrália, aos 2,4 anos de idade, plantado em Butiá, RS.

\section{MATERIAL E MÉTODOS}

O presente estudo foi desenvolvido no município de Butiá, RS localizado na região fisionômica natural do Estado do Rio Grande do Sul, denominada Serra do Sudeste (Escudo Rio-grandense), nas seguintes coordenadas geográficas: Latitude $30^{\circ} 07^{\prime} 12^{\prime \prime}$ Sul e Longitude $51^{\circ} 57^{\prime} 45^{\prime \prime}$ Oeste de Greenwich e altitude de $35 \mathrm{~m}$ s.n.m. Pelo sistema de classificação de Koeppen (MORENO, 1961), o clima da região é do tipo Cfa, subtropical. A temperatura média do mês de janeiro é $24^{\circ} \mathrm{C}$, a temperatura média do mês de julho é $13^{\circ} \mathrm{C}$ e a temperatura média anual entre $18-19^{\circ} \mathrm{C}$, a temperatura média das máximas no ano é de $24^{\circ} \mathrm{C}$ e a temperatura média das mínimas no ano é de $14^{\circ} \mathrm{C}$. A precipitação pluviométrica nos meses de janeiro e julho e a precipitação anual são, respectivamente, $120-140 \mathrm{~mm}, 120 \mathrm{~mm}$ e $1400 \mathrm{~mm}$ (IPAGRO, 1989).

Segundo BRASIL (1973), o solo da região em estudo pertence à Unidade de Mapeamento São Jerônimo; na classificação brasileira atual, é conhecido como Podzólico Vermelho Escuro, textura argilosa, relevo ondulado e substrato granito. (OLIVEIRA et al., 1992). Esta Unidade de Mapeamento é formada, na sua maior parte, por solos profundos, bem drenados, de coloração avermelhada, textura franco argilosa a argilosa com cascalhos, porosos e desenvolvimento a partir de granitos. Os solos desta Unidade são fortemente ácidos, com saturação e soma de bases baixas e teores baixos de matéria orgânica (BRASIL, 1973). 
A acácia-negra utilizada no presente estudo é procedente de Lake George Bunge Dore, New South Wales, Austrália, com as seguintes características geográficas: Latitude $35^{\circ} 28^{\prime}$ sul; Longitude $148^{\circ} 57^{\prime}$ leste.

A partir de uma distribuição diamétrica e utilizando-se duas equações de regressão $(\ln Y=a+b * \operatorname{lnDAP})$ para quantificar folhas, galhos vivos, casca e madeira, onde $\ln \mathrm{Y}=$ logaritmo natural do peso seco (biomassa) dos componentes, em kg; a e b= coeficientes do modelo; lnDAP= logaritmo natural do diâmetro à altura do peito (DAP), com casca, em $\mathrm{cm}$ e $\left(\ln Y=\mathrm{a}+\mathrm{b}^{*} \operatorname{lnDAP}+\right.$ $\mathrm{c}+\ln \mathrm{H})$ para quantificar os galhos mortos, onde $\ln \mathrm{Y}=$ logaritmo natural do peso seco (biomassa) dos componentes, em kg; a, b e c= coeficientes do modelo; $\operatorname{lnDAP}=\log$ aritmo natural do diâmetro à altura do peito (DAP), com casca, em $\mathrm{cm}$ e $\mathrm{lnH}=$ logaritmo natural da altura total, em m determinou-se a biomassa em cada componente (CALDEIRA, 1998). Na parte intermediária da copa das árvores, nos quatro pontos cardeais, foram coletadas folhas para análise nutricional em fevereiro de 1997. Os galhos foram separados do tronco e classificados como vivos (verdes) e mortos (secos). Dos galhos vivos foram coletadas todas as folhas.

A massa fresca total das folhas, dos galhos (vivos e mortos), casca e madeira do tronco das árvores amostradas, foram determinadas no campo. De cada componente (folhas, galhos, madeira e casca), foram retiradas amostras para determinação da massa fresca, no campo. Cada componente foi acondicionado em sacos plásticos, devidamente identificados. As amostras foram transferidas para sacos de papel e levadas à estufa com circulação de ar forçada, para posterior secagem a temperatura de 70 a $75^{\circ} \mathrm{C}$.

$\mathrm{O}$ tronco foi amostrado utilizando-se um disco de $5,0 \mathrm{~cm}$ de espessura, retirado na metade da altura total da árvore, conforme a metodologia proposta por YOUNG \& CARPENTER (1976). Deste disco, foi separada a casca da madeira.

As amostras secas de madeira e casca foram picadas para facilitar a moagem; enquanto que as demais amostras foram moídas em moinho do tipo Wiley e passadas na peneira com malha $1,0 \mathrm{~mm}$. Após retirou-se uma amostra de $50 \mathrm{~g}$ de cada componente para a determinação da concentração dos nutrientes na matéria seca.

As concentrações de nitrogênio, fósforo, potássio, cálcio, magnésio e enxofre foram determinadas de acordo com a metodologia proposta por TEDESCO et al. (1995).

$\mathrm{O}$ estoque dos nutrientes $\left(\mathrm{kg} \cdot \mathrm{ha}^{-1}\right)$ nos componentes da biomassa aérea foi obtido a partir da biomassa determinada por CALDEIRA (1998) e após a obtenção da concentração dos nutrientes determinada para cada componente (folhas, galhos vivos e mortos, madeira e casca). 


\section{RESULTADOS E DISCUSSÃO}

As folhas possuem as maiores concentrações de $\mathrm{N}, \mathrm{P}, \mathrm{K}, \mathrm{Mg}$ e S em relação aos outros componentes (Tabela 1). Comportamento semelhante foi observado por outros autores como TANDON et al. (1988) em plantações de Eucalyptus grandis, Austrália; GEORGE \& VARGHESE (1990) em Eucalyptus globulus; SCHUMACHER (1992) em Eucalyptus camaldulensis, E. grandis e E. torelliana; BAGGIO (1994) em Mimosa scabrella; VEZZANI (1997) em povoamentos puros e mistos de Eucalyptus saligna e Acacia mearnsii; CARBONERA PEREIRA et al. (1998) em Acacia mearnsii $e$ CALDEIRA (1998) em Acacia mearnsii. Contudo, nas folhas, as concentrações, principalmente de $\mathrm{N}$ são superiores àquelas encontradas por CAMPOS (1991) em Ilex paraguariensis; VETTORAZZO et al. (1993) em Eucalyptus grandis, E. camaldulensis e E. torelliana; COSTA et al. (1996) em Acacia mangium e AMARAL et al. (1996) em Dalbergia nigra. Portanto, isso comprova que as maiores concentrações de $\mathrm{N}$ nas folhas de Acacia mearnsii possuem um maior potencial de ciclagem de nutrientes desta espécie, via serapilheira, do que outras espécies como por exemplo, eucalipto. Pois, a concentração de nutrientes nas folhas das árvores é influenciada por diversos fatores como as condições de sítio, a idade das folhas, a posição das folhas na copa, época do ano (VAN DEN DRIESSCHE, 1984; BELLOTE, 1990; AMARAL, 1996) e também entre procedências (CALDEIRA, 1998).

$\mathrm{O}$ alto teor de $\mathrm{N}$ nas folhas de acácia-negra pode ser justificado pela capacidade que a mesma possui, como espécie leguminosa, de fixar $\mathrm{N}_{2}$ e pelo efeito sinérgico da ação da bactérias diazotróficas e micorrizas (FRANCO et al., 1992). Segundo AUER \& SILVA (1992), a acácia-negra, em solos tropicais, pode fixar até $200 \mathrm{~kg}$ de $\mathrm{N}$ ha. ano $^{-1}$.

Tabela 1 - Concentração de nutrientes nos diferentes componentes da biomassa aérea de Acacia mearnsii 2,4 anos de idade, em Butiá, RS

Table 1 - Concentration of nutrients in the different components of above-ground biomass of Acacia mearnsii, with 2.4 years old, municipality of Butiá, RS

\begin{tabular}{ccccccc}
\hline $\begin{array}{c}\text { Componentes } \\
\text { (Components) }\end{array}$ & \multicolumn{7}{c}{$\begin{array}{c}\text { Nutrientes }\left(\mathrm{g} \mathrm{kg}^{-1}\right) \\
\text { (Nutrients) }\end{array}$} \\
\cline { 2 - 7 } & $\mathbf{N}$ & $\mathrm{P}$ & $\mathrm{K}$ & $\mathrm{Ca}$ & $\mathrm{Mg}$ & $\mathbf{S}$ \\
\hline $\begin{array}{c}\text { Folhas } \\
\text { Leaves }\end{array}$ & 23,78 & 1,10 & 11,33 & 4,41 & 1,78 & 1,36 \\
$\begin{array}{c}\text { Galhos Vivos } \\
\text { Live Branches } \\
\text { Galhos Mortos } \\
\text { Dead Branches }\end{array}$ & 8,00 & 0,70 & 9,86 & 2,98 & 1,03 & 0,70 \\
$\quad \begin{array}{c}\text { Casca } \\
\text { Bark } \\
\begin{array}{c}\text { Madeira } \\
\text { Wood }\end{array}\end{array}$ & 10,31 & 0,44 & 7,10 & 7,30 & 1,47 & 0,60 \\
\hline
\end{tabular}


As concentrações de $\mathrm{N}$ e $\mathrm{Mg}$ nas folhas de Acacia mearnsii são similares àquelas encontradas por FROUFE et al. (1998) para Albizia guachapele, pois os maiores teores de nutrientes, especialmente de $\mathrm{N}$ encontrados nas folhas de Albizia guachapele, segundo o autor demonstram que esta espécie possui um maior potencial de ciclagem de nutrientes, via serapilheira. Contudo, as concentrações de $\mathrm{P}$ nas folhas de Acacia mearnsii são similares àquelas encontradas pelos autores acima em plantios puros de $E$. grandis e em plantios consorciados com Albizia guachapele e E. grandis.

A elevada concentração de nutrientes nas folhas, em relação a outros componentes torna este componente um maior potencial de ciclagem de nutrientes, embora represente um pequeno percentual em relação à biomassa total das árvores. Nas folhas, encontram-se a maioria das células vivas, que tendem a acumular maiores quantidades de nutrientes, em função dos processos de transpiração e fotossíntese (KRAMER \& KOZLOWSKI, 1979; KOZLOWSKI et al., 1991).

As concentrações de $\mathrm{P}$ e $\mathrm{K}$ nas folhas, no presente estudo (2,4 anos de idade) foram maiores do que um povoamento comercial com 3,0 anos de idade, exceto no caso do Ca (CALDEIRA et al., 1999a), porém as concentrações de $\mathrm{N}, \mathrm{P}$ e K nas folhas, no presente estudo, foram maiores do que uma procedência de Batemans Bay de acácia-negra com 3,0 anos de idade, enquanto que as concentrações de $\mathrm{Ca}$ e $\mathrm{Mg}$ foram menores no povoamento do presente estudo (CALDEIRA et al., 1999i).

$\mathrm{Na}$ acácia-negra deste estudo as maiores concentrações de $\mathrm{Ca}$ foram observadas na casca (Tabela 1). O cálcio é um elemento menos redistribuído na planta pelo fato de possuir uma baixa mobilidade no floema. Resultados semelhantes foram observados por PANDE et al. (1987) em Eucalyptus híbrido Acacia auriculiformis; CAMPOS (1991) em Ilex paraguariensis; LELES et al. (1995) em Eucalyptus camaldulensis e E. pellita; VEZZANI (1997), CARBONERA PEREIRA et al. (1998) e CALDEIRA (1998) em Acacia mearnsii. Conforme, ATTIWILL et al. (1978), o Ca está associado à lignificação das paredes celulares, não sendo redistribuído para as partes novas da planta.

No estudo realizado por CALDEIRA (1998), a procedência de Bodalla - Austrália (Acacia mearnsii), além de ter as maiores concentrações de Ca na casca também apresenta elevada concentração nas folhas. Este fato pode ser uma característica desta procedência, pois a concentração de nutrientes nos componentes da matéria seca arbórea pode estar relacionada com a produção de matéria seca aérea e subterrânea, com a espécie, solo e espaçamento.

Quanto ao $\mathrm{Mg}$, as maiores concentrações foram observadas nas folhas (Tabela 1). É comum que o Mg esteja em concentração mais elevada nas folhas, devido o mesmo fazer parte do componente fotossintético, ou seja, faz parte da estrutura da clorofila $a$ e $b$ (MAGALHÃES, 1979; KRAMER \& KOSLOWSKI, 1979; AWAD \& CASTRO, 1983; SALISBURY \& ROSS, 
1992; KOSLOWSKI, et al., 1991). Vários autores (TURNER, 1986; TANDON et al., 1988; TURVEY \& SMETHURST, 1994; DRUMOND et al., 1997; GOMES et al., 1997; CALDEIRA, 1998) também verificaram o mesmo em diferentes espécies e idades. As concentrações de $\mathrm{Ca}$ e $\mathrm{Mg}$ na folhas podem variar de acordo com a época do ano (KRAMER \& KOZLOWSKI (1979), com a idade das folhas e entre procedências (CALDEIRA, 1998; CARBONERA PEREIRA et al., 1998; CALDEIRA et al., 1999a; 1999i).

Tabela 2 - Quantidade média de nutrientes nos diferentes componentes da biomassa aérea de Acacia mearnsii, 2,4 anos de idade, em Butiá, RS

Table 2 - Medial amount of nutrients in the different components of above-ground biomass of Acacia mearnsii, with 2.4 years old, municipality of Butiá, $R S$
Componentes
B. Aérea
Nutrientes
(Components)
(Above-ground
(Nutrients)
biomass)

\begin{tabular}{|c|c|c|c|c|c|c|c|}
\hline & & \multicolumn{6}{|c|}{$\left(\mathrm{kg}^{\prime} \mathrm{ha}^{-1}\right)$} \\
\hline & & $\mathrm{N}$ & $\mathrm{P}$ & $\bar{K}$ & $\mathrm{Ca}$ & $\mathrm{Mg}$ & $S$ \\
\hline $\begin{array}{l}\text { FOLHAS } \\
\text { (Leaves) }\end{array}$ & 7486,40 & 178,03 & 11,58 & 84,82 & 33,01 & 14,40 & 10,18 \\
\hline $\begin{array}{l}\text { Galhos Vivos } \\
\text { (Live branches) }\end{array}$ & 8008,24 & 64,07 & 5,61 & 78,96 & 23,86 & 8,25 & 5,61 \\
\hline $\begin{array}{l}\text { Galhos Mortos } \\
\text { (Dead branches) }\end{array}$ & 513,60 & 2,81 & 0,10 & 2,33 & 1,93 & 0,58 & 0,15 \\
\hline $\begin{array}{l}\text { Casca } \\
\text { (Bark) }\end{array}$ & 4751,57 & 49,99 & 2,09 & 33,74 & 34,69 & 6,98 & 2,85 \\
\hline $\begin{array}{l}\text { Madeira } \\
(\text { Wood })\end{array}$ & 20346,99 & 39,47 & 4,27 & 59,82 & 14,24 & 4,88 & 2,64 \\
\hline Total & 41106,80 & 334,37 & 23,65 & 259,67 & 107,73 & 35,09 & 21,43 \\
\hline
\end{tabular}

A ordem das quantidades totais dos nutrientes é similar aos resultados encontrados por BAGGIO \& CARPANEZZI (1997b), com Mimosa scabrella. Contudo, CARBONERA PEREIRA et al. (1998) verificaram que em Acacia mearnsii, aos 9 anos de idade, as quantidades totais de todos os nutrientes na biomassa aérea teve a seguinte ordem: $\mathrm{N}>\mathrm{Ca}>\mathrm{K}>\mathrm{Mg}>\mathrm{P}$.

Dos nutrientes analisados por BAGGIO \& CARPANEZZI (1997b) em Mimosa scabrella, no sul do Brasil (N, P, K, Ca, Mg, S, Cu, Fe, Mn, Zn), $73,3 \%$ da quantidade total foi encontrada no lenho, contra $28,7 \%$ da copa.

A proporção de nutrientes nas folhas foi maior que nos demais componentes da biomassa aérea. As folhas acumularam em torno de 53\% do 
total de $\mathrm{N} ; 49 \%$ do total de $\mathrm{P}$ e $48 \%$ do total de S (Tabela 3), ainda que esta tenha participação percentual superior à das folhas no total da biomassa aérea (CALDEIRA, 1998).

Tabela 3 - Proporção de nutrientes acumulados nos componentes da biomassa aérea de Acacia mearnsii, 2,4 anos de idade, em Butiá, RS

Table 3 - Ratio between accumulated nutrients in the components of above-ground biomass, with 2.4 years old, municipality of Butiá, RS

\begin{tabular}{ccccccc}
\hline $\begin{array}{c}\text { Componentes } \\
\text { (Components) }\end{array}$ & \multicolumn{7}{c}{$\begin{array}{c}\text { Nutrientes (\%) } \\
\text { (Nutrients) }\end{array}$} \\
\cline { 2 - 7 } & $\mathrm{N}$ & $\mathrm{P}$ & $\mathrm{K}$ & $\mathrm{Ca}$ & $\mathrm{Mg}$ & $\mathrm{S}$ \\
\hline $\begin{array}{c}\text { FOLHAS } \\
\text { (Leaves) }\end{array}$ & 53,00 & 49,00 & 33,00 & 31,00 & 41,00 & 48,00 \\
$\begin{array}{c}\text { Galhos Vivos } \\
\text { (Live branches) } \\
\text { Galhos Mortos }\end{array}$ & 19,00 & 23,00 & 30,00 & 22,00 & 23,00 & 26,00 \\
$\begin{array}{c}\text { (Dead branches }) \\
\quad \text { Casca }\end{array}$ & 1,00 & 0,40 & 1,00 & 2,00 & 2,00 & 1,00 \\
$\begin{array}{c}\text { (Bark) } \\
\text { Madeira } \\
\text { (Wood) }\end{array}$ & 15,00 & 0,60 & 13,00 & 32,00 & 20,00 & 13,00 \\
\hline & 12,00 & 18,00 & 23,00 & 13,00 & 14,00 & 12,00 \\
\hline
\end{tabular}

A copa (folhas, galhos vivos e mortos) acumularam em média $67,6 \%$ do total de nutrientes, no entanto os $32,4 \%$ restantes foram acumulados no fuste (madeira e casca). Vários autores (REZENDE et al., 1983; SHARMA \& PANDE, 1989; REIS \& BARROS, 1990; CARBONERA PEREIRA et al., 1998; CALDEIRA, 1998) verificaram que, em povoamentos florestais, a copa contém em torno de $50 \%$ da quantidade total dos nutrientes. Isto é comprovado em função do que durante a fase juvenil de um povoamento florestal, uma grande parte da alocação dos carbohidratos são canalizados para a produção de biomassa da copa. No entanto, com o passar do tempo, quando as copas começam a competir entre si, a produção relativa do tronco aumenta e a das folhas e ramos diminui gradativamente (CROMER, et al., 1975; ANDRAE, 1982; LARCHER, 1984; FABIÃO, 1986; REIS \& BARROS, 1990; OTTO, 1994).

Considerando a subdivisão usual biomassa da copa/biomassa do tronco, constatou-se que $61,07 \%$ da matéria seca da acácia-negra foi alocada para folhas, galhos vivos e mortos, contendo $73 \%$ do N; $72,4 \%$ do $\mathrm{P}, 64 \%$ do $\mathrm{K}, 55 \%$ do $\mathrm{Ca}, 66 \%$ do $\mathrm{Mg}$ e $75 \%$ do $\mathrm{S}$ do total existente. O componente fuste (casca e madeira) acumulou $27 \%$ do N; $27,6 \%$ do P; $36 \%$ do $\mathrm{K}, 45 \%$ do $\mathrm{Ca}$, $34 \%$ do $\mathrm{Mg}$ e $25 \%$ do S (Tabela 3). Resultados similares foram observados por VEZZANI (1997) em acácia-negra, aos 3,7 anos de idade. Entretanto, nos galhos, esse autor encontrou proporções maiores do que os observados neste estudo. 


\section{CONCLUSÕES}

As maiores concentrações de nutrientes na acácia-negra, aos 2,4 anos de idade, foram observados nas folhas, com exceção do Ca na casca, pois o mesmo é exportado para fora do sítio com a retirada da madeira e casca.

As menores concentrações de $\mathrm{N}, \mathrm{K}, \mathrm{Ca}, \mathrm{Mg}$ e $\mathrm{S}$ estão na madeira e do $\mathrm{P}$ nos galhos mortos.

No povoamento de acácia-negra, as folhas, galhos vivos e mortos acumularam em média $67,6 \%$ do total de nutrientes, enquanto que madeira e casca acumularam os $32,4 \%$ restante.

Recomenda-se dar continuidade ao trabalho, ao longo da rotação do povoamento, com objetivo de quantificar o conteúdo de nutrientes em povoamentos mais velhos, para subsidiar o manejo da espécie.

\section{BIBLIOGRAFIA CITADA}

AMARAL, F.C.S.do; GRAÇA, P.M.L.A.; BRITO, M.M.P.; ABREU JUNIOR, C.H.de; CAMPOS, D.C. Comparação entre a concentração de nutrientes das folhas e do folhedo do jacaranda-da-bahia (Dalbergia nigra) e cabreúva (Myroxylom peruiferum). In: SOLO SUELO - CONGRESSO LATINO AMERICANO DE CIÊNCIA DO SOLO, 13, Águas de Lindóia, 1996. Resumos expandidos... Águas de Lindóia, 1996. Publicação em CD Rom.

ANDRAE, F.H. Zweitinventur eines Eucalyptus saligna Bestandes in Südbrasilien. Centralblatt für das Gesamte Forstwesen, Klosterneuburg, v,99, n.4, p.193-217, 1982.

ATTIWILL, P.M.; GUTHRIE, H.B.; LEUNING, R. Nutrient cycling in a Eucalyptus obliqua (L'Herit) forest. I. Litter production and nutrient return. Australian Journal of Botany, Melbourne, v.261, p.79-91, 1978.

AUER, C.G.; SILVA, R. Fixação de nitrogênio em espécies arbóreas. In: CARDOSO, E.J.B.N.; TSAI, S.M.; NEVES, M.C.P. Microbiologia do Solo. Campinas: Sociedade Brasileira de Ciência do Solo, 1992. p.157-172. AWAD, M.; CASTRO, P.R.C. Introdução à fisiologia vegetal. São Paulo: Nobel, 1983. 177p.

BAGGIO, A.J. Estudio sobre el sistema agroforestal tradicional de la bracatinga (Mimosa scabrella Benth.) en Brasil: Productividad, manejo de residuos y elaboracion de compost. Madrid: Universidade Politecnica de Madrid: Escuela Tecnica Superior de Ingenieros de Montes, 1994. 242p. Tese (Doutorado) - Universidade Politecnica de Madrid, 1994.

BAGGIO, A.J.; CARPANEZZI, A.A. Estoque de nutrientes nos resíduos da exploração de bracatingais. Boletim de Pesquisa Florestal, Colombo, n.34, p.17-29, $1997 \mathrm{~b}$. 
BELLOTE, A.F.J. Nahrelementversorgung und wuchsleistung von gedungten Eucalyptus grandis - plantagem im cerrado von São Paulo (Brasilien). Freiburg, 1990. 159p. (Doutorado) - Institut fur Bodenkunde und Waldernahrungslehre der Albert-Ludwigs, Universitat Freiburg, 1990.

BRASIL. Ministério da Agricultura. Departamento Nacional de Pesquisa Agropecuária. Levantamento de reconhecimento dos solos do Estado do Rio Grande do Sul. Recife, 1973. 431p. (Boletim Técnico, 30).

CALDEIRA, M.V.W. Quantificação da biomassa e do conteúdo de nutrientes em diferentes procedências de Acácia-negra (Acacia mearnsii De Wild.). Santa Maria-RS. 1998. 96p. Dissertação (Mestrado em Engenharia Florestal) - Curso de Pós-Graduação em Engenharia Florestal, Universidade Federal de Santa Maria, 1998.

CALDEIRA, M.V.W.; CARBONERA PEREIRA, J.; SCHUMACHER, M.V.; DELLA-FLORA, J.B.; SANTOS, E.M. Comparação entre as concentrações de nutrientes nas folhas e no folhedo em procedência de Acacia mearnsii De Wild. Revista Árvore, Viçosa, v.23, n.4, p.489-492, 1999i.

CALDEIRA, M.V.W.; SCHUMACHER, M.V.; CARBONERA PEREIRA, J.; DELLA-FLORA, J.B.; SANTOS, E.M. Concentração e redistribuição de nutrientes nas folhas e no folhedo em povoamento comercial de Acacia mearnsii De Wild. no Rio Grande do Sul. Ciência Florestal, Santa Maria, v.9, n.1, p.19-24, 1999a.

CAMPOS, M.A.A. Balanço de biomassa e nutrientes em povoamentos de Ilex paraguariensis. Avaliação na safra e na safrinha. Curitiba, 1991. 106p. Dissertação (Mestrado) - Universidade Federal do Paraná.

CARBONERA PEREIRA, J.; SCHUMACHER, M.V.; HOPPE, J.M.; CALDEIRA, M.V.W.; SANTOS, E.M. Determinação do conteúdo de nutrientes em um povoamento de Acacia mearnsii De Wild. no Rio Grande do Sul. Revista Árvore, Viçosa, 1998. (no prelo).

COSTA, G.S.; ANDRADE, A.G.de; FARIA, S.M. Ciclagem de nutrientes em um plantio de Acacia mangium com seis anos de idade. In: SOLO SUELO - CONGRESSO LATINO AMERICANO DE CIÊNCIA DO SOLO, 13, Águas de Lindóia, 1996. Resumos expandidos... Águas de Lindóia, 1996. Publicação em CD Rom.

CROMER, R.N.; RAUPACH, M.; CLARKE, A.R.P.; CAMERSON, J.N. Eucalyptus plantaions in Australia. The potencial for intensive production. Appita, p.165-173, 1975.

DRUMOND, M.A.; BARROS, N.F.; SOUZA, A.L.; SILVA, A.F. Distribuição de biomassa e de nutrientes em diferentes coberturas florestais e pastagem na região do médio Rio Doce-MG. Revista Árvore, v.21, n.2, p.187-199, 1997. 
FABIÃO, A.M.D. Contribuição para o estudo da dinâmica da biomassa e produtividade primária líquida em eucaliptais. Lisboa, Portugal. 1986.

Tese (Doutorado) - Instituto Superior de Agronomia, 1986.

FRANCO, A.A.; CAMPELLO, E.F.; SILVA, E.R.; FARIA, S;M. de. Revegetação de solos degradados, 1992. 9p. EMBRAPA - CNPAB (Comunicado Técnico, $\mathrm{n}^{\circ}$ 9).

FROUFE, L.C.M; FRANCO, A.A.; FARIA, S.M de; CAMPELLO, E.F.C. Reciclagem de nutrientes via folhas - serapilheira de Eucalyptus grandis e Albizia guachapele cultivados em sistemas puros e consorciados. FertBio 98: REUNIÃO BRASILEIRA DE FERTILIDADE DO SOLO E NUTRIÇÃO DE PLANTAS; 23; REUNIÃO BRASILEIRA SOBRE MICORRIZAS, 7; SIMPÓSIO BRASILEIRO DE MICROBIOLOGIA DO SOLO, 5; REUNIÃO BRASILEIRA DE BIOLOGIA DO SOLO, 2, Caxambú, 1998. Resumos expandidos... Caxambú, p.559, 863p.

GEORGE, M.; VARGHUESE, G.; Nutrient cycling in Eucalyptus globulus plantations. I Organic matter production, nutrients accumulation in standing crop and nutrients removal through harvest. Indian Forestry, v.116, n.1, p.42-48, 1990.

GOMES, F.S.; PESSOTTI, J.E.S.; PACHECO, R.M. Exportação de nutrientes por clones de Eucalyptus urophylla, em três unidades de solo no Vale do Rio Jari. In: IUFRO CONFERENCE ON SILVICULTURE AND IMPROVEMENT OF EUCALYPT, Salvador, 1997. Proceedings... Colombo: EMBRAPA. Centro Nacional de Pesquisa de Florestas, 1997, 3v. p.209-214.

IPAGRO - Instituto de Pesquisas Agronômicas. Seção de Ecologia Agrícola (Porto Alegre, RS). Atlas agroclimático do Estado do Rio Grande do Sul. Porto Alegre, 1989. 3v.

KIMMINS, J.P. Forest Ecology. New York: Collier Macmillan Canada, 1987. $531 \mathrm{p}$.

KOZLOWSKI, T.T; KRAMER, P.J.; PALLARDY, S.G. The physiology ecology of wood plants. San Diego, USA: Academic Press, 1991. 657p.

KRAMER, P.J.; KOZLOWSKI, T.T. Physiology of wood plants. New York: Academic Press, 1979. 811p.

LARCHER, W. Oekologie der pflanzen. Stuttgart: Eugen Ulme Gmbh \& Co, 1984. 320p.

LELES, P.S.S., REIS, G.G., REIS, M.G.F. Distribuição de nutrientes em Eucalyptus camaldulensis e Eucalyptus pellita sob diferentes espaçamentos, na região de cerrado. In: CONGRESSO BRASILEIRO DE CIÊNCIA DO SOLO, 25, 1995. Viçosa, MG. Resumos expandidos... Viçosa: Sociedade Brasileira de Ciência do Solo, 1995, v.2, p.862-863. $1158 \mathrm{p}$.

MAGALHÃES, A.C.N. Fotossíntese. In: FERRI, M.G. Fisiologia vegetal. São Paulo EDUSP, 1979. 350 p. p.117-163. 
MORENO, J. A. Clima do Rio Grande do Sul. Porto Alegre: Secretaria da Agricultura, 1961. 42p.

OLIVEIRA, J.B.; JACOMINE, P.K.; CAMARGO, M.N. Classes gerais de solos do Brasil. Jaboticabal, FUNEP, 1992. 201p.

OTTO, H.J. Waldoekologie. Stuttgart: Ulme, 1994. 391p.

PANDE, M.C.; TANDON, V.N.; SHANKER, P.P. Distribution of nutrients in age series of Eucalyptus and Acacia auriculiformis plantations in Bihar. Indian Forester, v.113, n.6, p.418-426, 1987.

POGGIANI, F. Alterações dos ciclos biogeoquímicos em florestas. In: CONGRESSO NACIONAL SOBRE ESSÊNCIAS NATIVAS, 2, 1992. São Paulo, SP. Anais... São Paulo: Revista do Instituto Florestal, 1992, v.3, p.734-739. 982p.

PRITCHETT, W.L. Suelos forestales: propiedades, conservación y mejoramiento. John Wiley of Sons, Inc. México, 1990. 634p.

REIS, M.G.F.; BARROS, N.F. Ciclagem de nutrientes em plantios de eucalipto. In: BARROS, N.F.; NOVAIS, R.F. de. eds. Relação soloeucalipto. Viçosa, Ed. Folha de Viçosa. 330p. p.265-301, 1990.

REZENDE, G.C.; BARROS, N.F.; MORAES, T.S.A.; MENDES, C.J. Produção e macronutrientes em florestas de eucalipto sob duas densidades de plantio. Revista Árvore, Viçosa, v.7, p.165-196, 1983.

RODIN, L.E.; BAZILEVICH, N.I. Production and mineral cycling in terrestrial vegetacion. Edimburgh: Oliver and Boyd, 1967.

SALISBURY, F.B.; ROSS, C.W. Plant physiology. Belmont, California: Wadsworth Publishing Company, 1992. 682p.

SCHUMACHER, M.V. Aspectos da ciclagem de nutrientes e do microclima em talhões de Eucalyptus Camaldulensis Dehnh, Eucalyptus grandis Hill ex Maiden e Eucalyptus torelliana F. Mesell. Piracicaba-SP. 1992. 87p. Dissertação (Mestrado em Ciências Florestais) - Escola Superior de Agricultura "Luiz de Queiroz" - Universidade de São Paulo, 1992.

SCHUMACHER, M.V. Ciclagem de nutrientes como base da produção sustentada em ecossistemas florestais. In: SIMPÓSIO SOBRE ECOSSISTEMAS NATURAIS DO MERCOSUL: O AMBIENTE DA FLORESTA, 1996. Santa Maria, RS. Anais ... Santa Maria: UFSM, CEPEF, 1996. p.65-77. 167p.

SHARMA, S.C.; PANDE, P.K. Patterns of litter nutrient concentrations in some plantation ecosystems. Forest Ecology and Management, v.29, p.151-163, 1989.

TANDON, V.N.; PANDE, M.C.; SINGH, R. Biomass estimation and distribution of nutrient in five different aged Eucalyptus grandis plantations in Kerala state. Indian Forester, v.114, n.4, p.184-199, 1988.

TEDESCO, M.J.; GIANELLO, C.; BISSANI, C.A.; BOHNEN, H.; VOLKWEISS, S.J.; Análise de solos, plantas e outros materiais. Porto 
Alegre: UFRGS, Departamento de Solos/Faculdade de Agronomia,1995. 174p. (Boletim Técnico, 5).

TURNER, J. Organic matter accumulation in a series of Eucalyptus grandis plantations. Forestry Ecology and Management, Amsterdam, v.17, n.2/3, p.231-242, 1986.

TURVEY, N.D.; SMETHURST, P.J.; Nutrient concentrations in foliage, litter and soil in relations to wood production of 7 to 15 year old Pinus radiata in Victoria, Austrália. Australian Forestry, Canberra, v.57, n.4, p.157-164, 1994.

VAN DEN DRIESSCHE, R. Prediction of mineral status of trees by foliar analysis. The Botanical Review, New York, v.40, p.347-394, 1984.

VETTORAZZO, S.C.; POGGIANI, F.; SCHUMACHER, M.V. Concentração e redistribuição de nutrientes nas folhas e no folhedo de três espécies de Eucalyptus. In: CONGRESSO FLORESTAL BRASILEIRO, 7, CONGRESSO FLORESTAL PANAMERICANO, 1, Curitiba, 1993. Anais... Curitiba, 1993, v.2, p.231-234.

VEZZANI, F.M. Aspectos nutricionais de povoamentos puros e mistos de Eucalyptus saligna (Smith) e Acacia mearnsii (De Wild.). Porto Alegre, RS. 1997. 97p. Dissertação (Mestrado em Ciência do Solo) - Universidade Federal do Rio Grande do Sul, 1997.

YOUNG, H.E.; CARPENTER, P.N. Sampling variation of nutrient element content within and between on trees of the same species. In: OSLO BIOMASS STUDIES, 1976. Oslo. Proceedings... Oslo, 1976. p.75-90.

\section{AGRADECIMENTOS}

À AGROSETA S.A., através do Engenheiro Florestal Elias Moreira dos Santos e aos funcionários, pelo apoio logístico, coleta de dados, material cedido e ao espaço físico. 